\section{Comment on "Selection of the Saffman-Taylor Finger Width in the Absence of Surface Tension: An Exact Result"}

Mineev-Weinstein has asserted [1] discovery of a selection principle for Saffman-Taylor fingers without surface tension. He begins with Saffman's exact timedependent finger solution,

$$
\begin{aligned}
z(t, \phi)= & \tau(t)+(2 \lambda-1) i \phi \\
& +2(1-\lambda) \log \left[e^{i \phi}-a(t)\right],
\end{aligned}
$$

mapping the lower half plane in $\phi$ to the fluid region. As $t \rightarrow \infty$, the pole location $a(t) \rightarrow 1$, and this solution approaches a steady finger of fractional width $\lambda$.

He considers perturbations that replace the $i \phi$ term by $\log \left(e^{i \phi}-\epsilon\right)$; if $\epsilon$ is small then this is a uniformly small perturbation on the interface. An exact solution may be written in which $\epsilon(t)$ depends on time but the coefficients are constant. As $t \rightarrow \infty, \epsilon(t) \rightarrow 1$ and the two logarithms merge, giving a steady finger of width $\lambda^{\prime}=\frac{1}{2}$. This "pole dynamics" and the exact solutions for fingers that change width have long been known [2].

That this class of solutions does not express any selection mechanism may easily be seen by the following argument: It is equally valid to replace only a fraction $\beta$ of the $i \phi$ term, changing

$$
i \phi \rightarrow(1-\beta) i \phi+\beta \log \left(e^{i \phi}-\epsilon\right) .
$$

As $t \rightarrow \infty$, the solution with this perturbed initial data will tend to an asymptotic finger of width $\lambda^{\prime}=\lambda-\beta(\lambda-$ $\frac{1}{2}$ ). The special case $\beta=1, \lambda^{\prime}=\frac{1}{2}$, is distinguished by vanishing of the coefficient multiplying $i \phi$, but presence or absence of an analytic term has no particular significance.

Mineev-Weinstein further considers replacing $i \phi$ by $\sum_{k=1}^{N} \delta_{k} \log \left(e^{i \phi}-\varepsilon_{k}\right)$ with $\sum \delta_{k}=1$; since all $\epsilon_{k} \rightarrow 1$ as $t \rightarrow \infty$, this perturbation also leads to a finger with $\lambda=\frac{1}{2}$. But just as above, these perturbations are a very special subclass. Even ignoring the equally dense set of perturbations for which singularity occurs in an arbitrarily short time, Mineev-Weinstein's claim that the finger with $\lambda=\frac{1}{2}$ is a nonlinear attractor is meaningless.

There is a wide variety of evidence to support the essential role of singular perturbations such as surface tension in finger width selection. If surface energy is anisotropic, then as its magnitude vanishes the limiting finger widths can be very narrow [3], very wide [4], or very complicated [5]. Thin film effects of vanishingly small magnitude select fingers of width different than $\frac{1}{2}$ [6]. Detailed analysis [7] has shown that arbitrarily small surface tension can have important effects over orderone times even for solutions with small curvatures. All of these results contradict Mineev-Weinstein's claim that finger width selection can be explained in a model without surface tension.

Robert F. Almgren

Department of Mathematics, The University of Chicago 5734 S. University Avenue

Chicago, Illinois 60637

Received 8 April 1998 [S0031-9007(98)07997-6]

PACS numbers: 47.15.Hg, 47.20.Hw, 68.10.-m, 68.70.+w

[1] M. Mineev-Weinstein, Phys. Rev. Lett. 80, 2113 (1998).

[2] S. D. Howison, J. Fluid Mech. 167, 439 (1986).

[3] D. A. Kessler, J. Koplik, and H. Levine, Phys. Rev. A 34, 4980 (1986).

[4] M. Ben Amar, R. Combescot, and Y. Couder, Phys. Rev. Lett. 70, 3047 (1993).

[5] R. Almgren, SIAM J. Appl. Math. 55, 1511 (1995).

[6] S. Tanveer, Proc. R. Soc. London A 428, 511 (1990).

[7] M. Siegel, S. Tanveer, and W.-S. Dai, J. Fluid Mech. 323, 201 (1996). 